$-1$

I.A-UR - 77-2063

TITLE:

CDNF-770925--

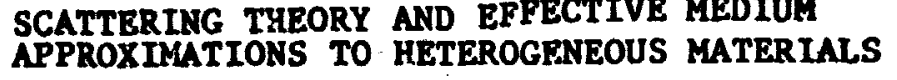

AUTHOR(B): $\quad$ J. E. Gubernatis

SUBMITTED TO: Conference on Electrical Transport and Optical Properties of

Inhomogeneous Media, September 7-9, 1977, at The Ohio State University, Columbus, Ohio.

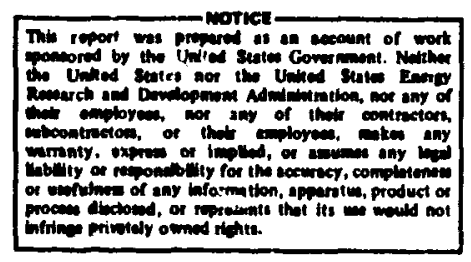

By acouptenos of this article for publication, the publisher recostrites the Government's (license) rights in any copyricht and tho Governmont and its authortsod ropresentative have warentrioted right to roproduce in whole or in part anld articls under any copyright weoured by the publisher.

The Los Alemow Soleatino Laboratory requents that the publisfier ldentify thib article ese worts porformed under the ausploes of the USERDA.

\title{
los Nalamos
}

\section{solentilic laboratory}

of the University of California

LOS ALAMOS, NEW MEXICO 87840

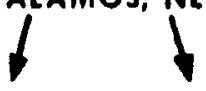

An Alfirmative Action/Equal Opporlunily Employer OISTRIRUTION OF THIS DOCIUMENT IS UNLIMITED

Form Nis. 8:1

$\operatorname{Sit}_{i \rightarrow 5} \sin 2820$
UNTTED STATES

ENERGY RESPARCH AND

DEVELOPMENT ADMENISTRATTON

CONTRACT W.7406.ENG. 36 
SCATTERING THEORY AND EFFECTIVE MEDIUM

APPROXIMATIONS TO HETEROGENEOUS MATERIALS

J. E. Gubernatis

Los Alamos Scientific Laboratory, Los 6. T.amos, NM 87545

\section{ABSTRACT}

The formal analogy existing between problems studied in the microscopic theory of disordered alloys and problens concerned with the effective (macroscopic) behavior of heterogeneous materials is discussed. Attention is focused on 1) analogous approximations (effective medium approximations) developed for the microscopic problems by scattering theory concepts and techniques, but for the macroscopic probler.s principally by intuitive means, 2) the link, provided by scattering theory, of the intuitively developed approxtmations to a well-defined perturbative analysis, 3) the possible presence of conditionally convergent integrals in effective medium approximations.

\section{INTRODUCTION}

Many phystcal phenomena are associated with inhomogeneous medium. For example, a solid state rhysicist may study the electrical properties of disordered alloys; a geologist, the mechanical properties of polycrystalline rocks. Other scientists may ponder the twinkling of stars or water-seepage through concrete.

Various theoretical approaches to these diverse phenomena generally exhibit two common features. One feature is a statement of physics, usually in the context of a model. This statement may be the equation of motion for a solid continuum, the time independent Schrödinger equation, Helmholtz's equation, etc. These various equations regulate the dynamical variables and have as parameters stochastic variables associated with the inhomogeneous medium. The second common feature is an average of the dynamical variables or their products over the distribution of the stochastic parameters. Even when the statistical information is complete, only an approximation to the averaging, which for some problems is called an effective medium approximation, is generally possible. Most often effective medium npproximations are developed by intultive means.

In solid state physics in the study of disordered alloys, theorists stated the physics of their problem in terms of an integral equation and analyzed this equation by techniques developed in the quantum mechanical theory of scattering. This integral equation is equivalent to a pecturbation series (an infinite series), and vartous effective medium approximations were developed usually by approximating the average of each term in the series and then summing an infinite series of terms. This approach, scattering theor, ith infintte order perturbation summation; developed perturbatively scveral effective medium approximations. Some of 
these approximations have direct analogs to approximations developed intuitively for other phenomena.

. I will illustrate the application of the scattering theory approach to the computation of the ef fective dielectric constant of a poiycrystal. I will state the problem in the form of an integral equation, recover several well-known intuitive approximatiuns, and indicate, but net demonstrate, the connection of the approximations to perturbation theory. This example and its discussion hopefully ciarify the meaning of several commonly used effective medium approximations.

For this same probletn, I will indicate the existence of conditiofally convergent terms that may appear in an improperly constituted perturbation serles. These terms can cause the effective property or approximations to the effective property of the material to depend on the shape of the material. Batchelor ${ }^{1-3}$ first ncticed the fossibllity of such terns in an analogous problem and suggested a procedure for their removal. His procedure is discussed in light of standard practices in scattering theory.

\section{STATEMENT OF THE PROBLEM}

The example under consideration is a polycrystalline material that has a dielectric constant tensor $\underline{\epsilon}(\underline{r})$ which in general changes from grain to grain because of changes in materfal type, orientation of the grain, or grain size and shape. However, when measured, the meterlal as a whole behaves effectlvely as a honogeneous medium with a dielectric constant $\underline{\epsilon}^{*}$. The problem is to calculate $\underline{\epsilon}^{*}$ from statistical information about $\underline{G}(\underline{r})$.

For a static problem the physics follows from

$$
\underline{\nabla} \cdot \underline{D}=0
$$

where $D$ is the electric displacement field. Modelling of the medium begins with the consitutive relation

$$
\underline{D}(\underline{r})=\underline{\underline{e}}(\underline{r}) \underline{E}(\underline{r})
$$

where $\underline{E}$ is the electric fleld, and the effective dielectric constant is defined by

$$
\lfloor\underline{D}\rangle=\underline{e}^{*}\langle\underline{E}\rangle
$$

where the angular brackets denote ensemble averaging.

The problem specifted by (1)-(3) is analogous to many other problems. As $E$ is a gradient of a potential, replacting $E$ by the gradient of temperature and $\underline{D}$ by the heat current re-interprets $E$ as a thermal conductivity. Through sinilar re-interpretations the problems of effective electrical conductivily, permeability, diffusivity, elastic stiffness, etc. are seen as directly analogous. The problem of effective elastic properties, of course, requires increases in tensorlal rank.

It is useful to write $e$ as the sum of two parts 


$$
e=\varepsilon^{\circ}+\delta e
$$

where $\varepsilon^{0}$ is some arbitrarily, but in general conveniently, chosen spatialiy Invariant (homogeneous) dielectric constant so that all stochastic variations are contained in the perturbation de. One can now show the equivalence of $(1)-(4)$ to the integral equation

$$
\underline{E}-\underline{E}^{\bullet}+\int d \underline{r}^{\prime}\left[\underline{\delta e}\left(\underline{\underline{r}}^{\prime}\right) \underline{\mathrm{E}}\left(\underline{\underline{r}}^{\prime}\right) \cdot \nabla^{\prime} \lg \left(\underline{\boldsymbol{r}}, \underline{\underline{x}}^{\prime}\right)\right.
$$

where $g$ is the Green's function satisfying

$$
\underline{\nabla} \cdot \underline{\underline{e}}^{0} \boldsymbol{g}=\delta(\underline{r}-\underline{\underline{\prime}})
$$

and $E^{\circ}$ satisfles the homogeneous equation

$$
\underline{\nabla} \cdot \underline{E}^{0} \underline{E}^{0}=0
$$

It is also useful to write the integral equation in several different forms. First, with a otandard Indiclal notation

$$
\underline{E}_{I}=E_{I}+\int_{\underline{d} x^{\prime}} G_{I j} \delta e_{j k} E_{k}
$$

where

$$
G_{1 j}=G_{j t}-B_{1,1}=\phi_{, 1 j}
$$

since $g_{1}$ is expresstbile as the gradient of a potential $\phi$. Next, in an operator notation

$$
E=E^{0}+G \delta E B
$$

where $G$ is an Integral operator

$$
G_{t} \rightarrow \int d \underline{r}^{\prime} G_{1 j}\left(\underline{r}, \underline{r}^{\prime}\right) f_{j}\left(\underline{\underline{r}}^{\prime}\right)
$$

Equation (6) Is a statement of the physics that is identical to (1)-(3).

The Integral equation has a formal solution obtained by iteration and represented by the infinite sertes

$$
\mathrm{E}=\mathrm{E}^{\circ}+\mathrm{G} \delta \epsilon \mathrm{E}^{\circ}+\mathrm{G} \delta \epsilon \mathrm{G} \delta \mathrm{E}^{\circ}+\cdots
$$

Different terms contain the perturbation $\delta \in$ to different "powers". With the definition of $T$, the T-matr.Lx,

$$
T=\delta E+\delta \epsilon G \delta E+\delta E G \delta \epsilon G \delta \epsilon+\cdots
$$

the following equivalent to (6) is obtained

$$
E=E^{\circ}+\text { GTE }^{\circ}
$$

Now $T$ represents the perturbation sertes, and a formal sumantion of 


$$
T=\delta E(I-G \delta E)^{-1}
$$

Since the explicit character of $\delta e$ is yet unspecifled, (6) and (7) axe general. The spectfication oi se for a polycrystal assumes the following model:

$$
\delta \varepsilon \underline{(\underline{r})}=\sum_{\alpha} \delta e^{\alpha} \theta^{\alpha}(\underline{r})
$$

where $\theta^{\alpha}(\underline{x})=1, \underline{x}$ in grain $\alpha$

$$
0 \text {, otherwise. }
$$

The term "grain" is used in the broadest possible sense. For example, the region $\alpha$ could be a pore.

To correspond to the piecewise behavior of $\delta E$, it is convenient to define a t-matrix associated with grain $\alpha$

$$
t^{\alpha}=\delta e^{\alpha} \theta^{\alpha}\left(I-G \delta e^{\alpha} \theta^{\alpha}\right)^{-1}
$$

With this definition $T$ is expressible as

$$
I=\sum_{\alpha} t^{\alpha}+\sum_{\alpha} \sum_{\beta \neq \alpha} t^{\alpha} G t^{\beta}+\sum_{\alpha} \sum_{\beta \neq \alpha} \sum_{\gamma \neq \beta} t^{\alpha} G^{\beta} G^{\beta}+\cdots
$$

Although (10) is equivalent to (7), it shifts the focus of the perturbation series from $\delta e$ to $t^{\alpha}$. The significance is that a truncation of (7) after the first term produces a perturbation series of first order in $\delta e^{\alpha}$, a truncation of (10) after the first term produces a perturbation of infinite order in $\delta \alpha$.

$\mathrm{t}^{\alpha}$ has a physical meaning: If the deviation from homogeneity is confined solely to one region $\alpha$, then

$$
E=E^{\circ}+G_{\delta}^{\alpha} \sigma_{E}^{\alpha}
$$

which when iterated and sumned becomes

$$
E=E^{\circ}+G t^{\alpha} E^{\circ}
$$

Thus $t^{\alpha}$ is the T-matrix which solves the single inholnogeneity problem. Consequently, the first term in (10) represents contributions from regions $\alpha$ individually embedded in a homogeneous medium $\epsilon^{\circ}$. The remaining term:; all involving at least two reglons, represent the interaction between regions.

\section{THE AVERAGTNG}

To compute $\epsilon^{*},\langle E\rangle$ and $\langle D\rangle$ are needed. $\langle E\rangle$ is determined directly from (7)

$$
\langle E\rangle=E^{\circ}+\left\langle(j T\rangle E^{\circ}\right.
$$


but the determination of $\langle D\rangle$ Involves several steps. First, since $\epsilon=e^{\circ}+\delta e$,

$$
\mathrm{D}=e^{\circ} \mathrm{E}+\delta e \mathrm{E}
$$

Next from a comparison of (6) and (7),

$$
\operatorname{SEB}=\mathrm{TE}^{\circ}
$$

Finally,

$$
\text { (D) }=e^{\circ}\langle\mathrm{E}\rangle+\langle\mathrm{T}\rangle \mathrm{E}^{\circ}
$$

Thus, (11) and (12) with (3) yield

$$
e^{*}=e^{0}+\langle T\rangle(I+\langle G T\rangle)^{-1}
$$

This is an exact equation, independent of the assumed polycrystalIine model.

As with most exact equations, exa:t evaluation is usualj.y impossible. Approximations are needed. In the present case, one oees that approximations to $T$ are espectally important, and one possible approximation is to truncate (10) after the first term.

$$
T \approx \sum_{\alpha} t^{\alpha}
$$

For the atandard problem of lootropic $\delta e^{\alpha}$ and spherical grains, the above, when used in (13), yields after several algebraic manipulations

$$
\frac{e^{*}-e^{0}}{e^{*}+2 e^{0}}=\sum v_{j} \frac{e^{J}-e^{0}}{e^{J}+2 e^{0}}
$$

where $v_{f}$ is the volume fraction of material type $j$ that his a dielectric constant $e \mathfrak{j}$. Often, a polarizability

$$
\frac{4 \pi \alpha^{J}}{3}-\frac{\epsilon^{J}-e^{\circ}}{e^{J}+2 e^{\circ}}
$$

Is assoclated with each grain, and (15) Is written as

$$
\frac{e^{*}-e^{0}}{e^{\star}+2 \epsilon^{0}}=\frac{4 \pi}{3} \sum_{j} v_{j} \alpha^{j}
$$

Th1s equation is now of the form of the tamous Clauglus-Mossott equation.

It 1s Importint to note that (14) 18 used in (13), not in

$$
e^{*}=e^{0}+(\mathrm{T})
$$


Since $t^{a}$ solves the probjen of a single grain a ambedded in $e^{\circ}$, (17) It a efmple ain of the average contribution of each grain: From elementary electrostatics, when in isotropic dielectric sphere is placed in a uniform electric field $B^{\circ}$, the ophere 18 polarlzed; to is connected with this polarization. Equation (14) sums Individual "dipoles" enbedded in $e^{\circ}$ and does not account for the fact that any given dipole sees a mediun in which-other "dipoles". are present. The factor $(I+(G T))^{-1}$ in $(13)$ is the Lorentz correction which accounts for the presence and the polarization of other grains by replacing these graine by a uniformly polarized medium. The Important point is that in (16) the grain a to embedded not in ". as In (14), but in a uniformly "polarized" medium. However, Interactions between grains are st11l neglected.

The approxtima:Ion (14) has been, used In any different contexts usually with $\left.e^{\circ}-\langle\rangle\right\rangle$, for example, in electrical conductivity prob-: lèns by Maxwe11 4,6 (frequency dependent) dielectric problems, by Maxwell-Garnett ${ }^{5 ; 6}$, thermal conductivity problem by deVries, and in solid state physics by Elliott and Taylor ${ }^{8}$. For elastic problems, Rröner' 8 result bears some relation to (17). In solid state physics, (14) is sometimes called the Average T-matrix Approximation (ATA).

A commonly used approximation is a self-consistent effective medium approximation. "Self-consistency" is a term used in different contexts, often incompatibly. In the present context, the gelf-consistency means the following: $T$ in (13) depends on $e^{\circ}$ through $\delta E$ and $G$. If $e^{0}$ were chosen so that $\langle T\rangle=0$, then $e^{*}=e^{0}$.

$T$. let alone $\langle T\rangle$, is not a quantity one e:pects to evaluate execti. Self-consistent solutions, however, san be sought for approximations to T. If, for example, one tal.es

$$
\mathrm{T} \simeq \sum_{\alpha} t^{\alpha}
$$

und requires

$$
\langle\mathrm{T}\rangle=0
$$

then for the standard problem of lsotropic $\delta^{\alpha}$ and opherical grains, he finds the following well-known self-consistent effective medium approximation

$$
\sum v_{j} \frac{e^{\star}-e^{j}}{e^{\star}+2 e^{j}}=0
$$

The self-consistency condition li gignificant. Both the ATA and self-consistent epproximation are based on a perturbation series represented by

$$
T \propto \sum_{\alpha} t^{\alpha}
$$


1.e., the embedding of single grains in a homogeneous medium. Equation (16), however, does not equel (19). The difference is the ATA stops with the above and thereby Ignores Interactions between grains, while the self-consistent approximation through the additional condition (18b) Incorporates Interacting grains in an average manner.

The mathematical details of how (18b) incorporates some of the interactions is fully documented elsewhere.10,11 Physically, one considers a single grain a in a unfformly polarized medium that represents the presence of the other grains. This polarized medium polarizes $\alpha$, but the polarization of $\alpha$ changes the polarization of the unifori medium, the change in this polarization changes the polarization of $\alpha$, etc. until the polarization of $\alpha$ and the uniform medium representing the other grains are consistent on the average. Self-consistent approximations, as defined in the present con, text, have been used by various investigators, for example ${ }^{\mathrm{Brugge}}$ ${ }^{\operatorname{man}} 12$ and Landauer ${ }^{13}$ for electrical media, Hershey ${ }^{14}$, H111 15 , and Budiansky 16 for elastic media. In solid state physics this approximation was derived by Taylor ${ }^{17}$ and by Soven 18 and is called the (single oite) Coherent Potential Approximation (CPA).

\section{REMARRS}

Fron (10) corrections to $\epsilon^{*}$ beyond the ATA are easily seen to be second order in $t$. Corrections to $e^{*}$ beyond the CPA are not as obvious, but are known to be fourth order in $t$, which implies that the ATA is an approximation to the CPA. The important point is that analysis natural to the scattering theory with infintte order perturbation summation approach can connect condition (18) to a well-defined perturbation analysis and identify the next order corrections: The CPA is exact through the third order in $t$. Since the self-consigtency conditioil is a formal expression of intultive and physical conditions stated for a variety of related problems, the ad hoc flavor of many self-consistent effective medium approximations is placed on cl-arly defined theoretical grounds by their relation to an explicit perturbation series that has immediately Identiflable; corrections.

On physical grounds the approximation,

$$
T=\sum_{\alpha} t^{\alpha}
$$

because it neglects interactions between grains and r.lustering effects is expected to work for well-separated grains of different spectes embedded In $e^{\circ}$. For two-phase syotems, this means small concentrations of one specles hosted in the othar. Because the CPA is alsu based on this same approximation to. $T$, it too is often regarded as a small concentration approximation. Th1s ts not corpletely correct alnce comparisons with experiment ouggest otheritse. Apparent1y, for many problems the Interactions between grains and such clusterlng effects as "touching" graing are on the average not as important as one would a prior1 suppose. 
Ior a tro-phase adiun the CPA Is exact for the low concentration linte for elther opecies. One interpretation of the CPA Is that of an Interpolation formula between these limita, an interpolation performod according to a secific approxination. The interpretation, of course, is not always useful for problems involving porous anterials.

Another important point about the CPA 1s that it is the best posalble approxtmation using etatiotical information about single gratnis, 0.8. volume fraction, texture, etc. It ls the best possible In the sense that it evaluates every perturbation tern that can be eraluated wich only single grain distrihution functions. Corrections to the CPA require knowledge of two-grain distribution functions. To be precise, the CPA 1s the beat aingle site approxtination in the sense that all oingle-site diagrams are taken into diccount.

An interesting, new effective medium approximation, called the reif-conpliatent curulant epproximation (SCA), was recently proposed by Hort and Yonezawa. 19-25 When they compared the SCA to the results of a computer experiment on a network of reelstors randouly placed on Bravals lattice, they found that near the percolatio.r 1imt the SCA matched the computed resulte better than the .PA givin In (19). Since clustering phenomena becomes important near the: per'olation threshold and since the CPA Igtores clugtering, It wculd appear that the SCA Includes some clustering effects. Indeed, Horl and Yonezawa 22 identify these contributions.

The SCA, however, 1s based on a special model off a heterogeneous material, called the perfectly disordered materiul. In. this. model the value of the dielectric constant at: $\underline{I}$ is stitistcally independent of the value at ${\underline{x^{\prime}}}^{\prime}$ ' (In a polycrystal, the values of $\delta$ at different points within each grain are strongly correlated; hence, a polycrystal does not fit the above definition of a pirfectly disordered material.) Additionally, the SCA also based on a infinite oumation of a perturbation serles that lncludes various terms in the aerles more than once. In the jargon of scattering: theory, the propagator is not renormalized in a self-consistent fashion so different diagrams are multiply counted. The physical and mathematical besis of the SCA needs further atudy.

There is more than one way to relate effective mealium approximations to perturbation theory. Elliott et $\mathrm{al} 10$ and Yoriezawa and Morigakili review these ways for solid otate physics problems. In a oeries of papers $19-25$ and with a opecific approach, Hori ant Yonezawa do an extensive analyais of electrical, thermal, and magnetic problenis. Gubernatis and Rrumhans ${ }^{26,27}$ discuss the analys1:3 as applied to elastic problems. Other references $28-40$ treat a variety of problems both from the intultive and more formal viewpoints. In some cases comparisons with experiment are given. This list is not close to being definitive.

\section{CONDITIONALLY CONVERGENT INTEGRALS}

Several years ago, Batchelor ${ }^{1-3}$ noted that if one attempts to calculate the effective properties of a hoterogeneous material by looking for a solution in terus of an expansion of the volume 
fraction $v$ of particles in a "dilute suspension", integrals that are only conditionally convergent can arise. If such integrals are evaluated for a finite volume which is allowed to go to infinity without change of shape, then the limit depends on the shape of the volume chosen. 41,42 Thus, the appearance of such integrals seemingly makes the effective property dependent on the shape of the sample. In the effective dielectric problem discussed above, conditionally convergent integrals with intagrands $Q\left(r^{-3}\right)$ arise from the dipole character of the $G_{i j}$ in $(6 a)$,

$$
G_{i j}=\frac{1}{4 \pi \epsilon^{\circ}} \frac{\partial}{\partial x_{i}} \frac{\partial}{\partial x_{j}} \frac{1}{\mid \underline{\underline{r}}-\underline{r}^{\prime} T}
$$

(the $1^{\text {th }}$ component of an electric field at $x$ because of the $j^{\text {th }}$ component of dipole placed at $\underline{\underline{r}}^{\prime}$ in a medium with dielectric constant $\left.\epsilon^{\circ}\right)$. To be properly convergent, the integrand at large $r$ must vary as $\mathrm{r}^{-(3+n)}$ where $n>0$.

Batchelor suggested a procedure ${ }^{i-3,41-3}$ to circumvent the apparent difficulties to $O\left(v^{2}\right)$. (If $v$ is small, terms $O\left(v^{2}\right)$ exhibit the first order effects of interaction between two particles.) Qualitatively, his procedure is as follows: Subtract from the given conditionally convergent integral another conditionally convergent integral whose integrand has the same asymtopic behavior as the integrand of the integral in question and whose ensemble average is zero. Then the ensemble average of the given integral is unchanged, but the troublesome asymtopic behavior of the integrand is removed. The result is now shape independent.

The source of difficulty is the long-ranged nature of the dipole field. Traditionally, in scattering theory (and many-body theory), long-ranged interactions are replaced by shorter-ranged screened interactions obtained by a partial, but infinite, sumation of perturbation series. Possibly, such a renormalization is related to Batchelor's intultive approach and would be a systematic way to generalize and extend his procedure.

A question of more immediate interest is whether the effective constant defined by (13) is we11-defined, 1.e. shape independent. I believe it is.

Briefly, relying heavily on results of Yonezawa and coworkers 11,22 , I reason in the following manner: First, Hrite ${ }^{\star}\left(G^{\prime \prime} T\right)=G\langle T\rangle$, then from (13) define $\epsilon^{*} \equiv \epsilon^{\circ}+\Sigma$ where

$$
\Sigma=\langle\mathrm{T}\rangle(\mathrm{I}+\mathrm{G}\langle\mathrm{T}\rangle)^{-1}
$$

is analogous to the self-energy that appears in the solid state physics of disordered alloys. One has that

\footnotetext{
*Although $\mathrm{T}$ contains all the statistical information, I prefer, because of $G$ being an integral operator, to write the average of GT as 'GT' to Indicate that the integration GT is averaged and not that the averaged $T$ is Integrated. Convention, along with Yonezawa and coworkers, uses $\mathrm{G}\langle\mathrm{T}\rangle$.
} 


$$
\Sigma=\Sigma^{(1)}+\Sigma^{(2)}+\Sigma^{(3)}+\cdots \cdot
$$

where

$$
\begin{aligned}
& \Sigma^{(1)}-(\delta e) \\
& \Sigma^{(2)}=\langle\delta E G E)-\langle\delta E(G(\delta E)
\end{aligned}
$$

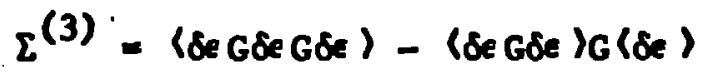

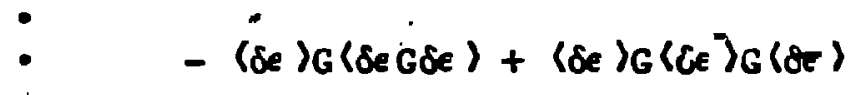

or in terms of cumulants

$$
\begin{aligned}
& \Sigma^{(1)}-\langle\delta e\rangle_{c} \\
& \Sigma^{(2)}=\langle\delta e G \delta\rangle_{c} \\
& \Sigma^{(3)}=\langle\delta e G \delta G \delta e\rangle_{c}-\left\langle\delta e G(\delta e\rangle_{c} G \delta e\right\rangle_{c} \\
& \vdots
\end{aligned}
$$

where (. - - / denotes tha cumulant. Equation (23) is composed of both reductble and irreducible terms where the "irreductble" has the ugual meaning: A diagram that cannot be divided into two separate parts just by cutting the propagator (the Green's function) once. (Yonezawa called these proper diagrams.) in the other hand, (24) Ls composed of only irreductble terms.

Only the reducible diagrams, however, can be conditionally convergent. The integrands of reductble terms are the product of $G$ times a function which may tend to a constant as $r \rightarrow \infty$ leaving an integrand $O\left(r^{-3}\right)$ and the integral conditionally convergent. This remark 1a exemplified by (23b): If the $\delta$ refer to different grains, ( $\delta e G \delta$ ) is reducible, and the principal contribution to the term 18 the Integration

$$
\iint \underline{d r}^{\prime} \underline{r}^{\prime} G\left(\underline{r}, \underline{r}^{\prime}\right) P_{2}\left(\underline{r}, \underline{r}^{\prime}\right)
$$

where $P_{2}\left(\underline{x}, \underline{x}^{\prime}\right.$ : is the probability of finding a grain centered at $\underline{x}$

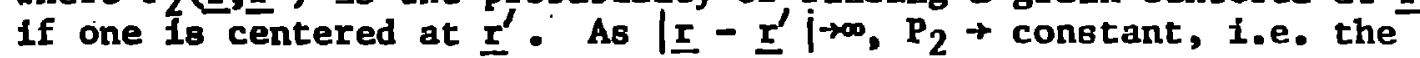
grains become statiaticaliy independent, and the integrand $1_{8}$ $O\left(r^{-3}\right)$. If the $\delta E$ refer to the same grain, ( $\delta e G \delta$ ) is irreducible, and the finite gize of the grain eliminates any question of conditional convergence. The second part of (23b), $(\delta e) G\langle\delta e\rangle$, is also conditionally convergent; the $\delta e$ 's are averaged iadependently and the integration involves simply the bare G with $\delta \epsilon$.

The two terms in (23b) combine to give (24b) which is irreducible and absolutely convergent. Equation (24b) is proportional to

$$
\iint \underline{d r d \underline{r}^{\prime}} G\left(\underline{r}, \underline{r}^{\prime}\right)\left[P_{2}\left(\underline{r}, \underline{r}^{\prime}\right)-P_{1}(\underline{r}) P_{1}\left(\underline{r}^{\prime}\right)\right]
$$


where $P_{f}(\underline{x})=v$ is the one-point correlation function. As $\mid \underline{x}-\underline{\underline{r}}^{\prime} f \stackrel{(x)}{\rightarrow}$

$$
\mathrm{P}_{2}\left(\underline{r}, \underline{\underline{r}}^{\prime}\right)-\mathrm{P}_{1}(\underline{\underline{r}}) \mathrm{P}_{1}\left(\underline{x}^{\prime}\right) \rightarrow 0
$$

and the integral is well-behaved since for any physicaily reasonable specification of $P_{2}$, It now goes to zero at leagt as fast as $r^{-(}(3+n)$ where $n>0$. A simple and, In the present context, an Important property of cumulants is illustrated: Contributions from statistically Independent stochastic varlables are not present.

Irreductbility with the non-local character of the Green's function Imply another mechanism to produce properly convergent integrals. A general character of Irreducibility is convoluted multiple integrals. In individual terms or portions of such terms Involving multiple integrations, a given integrand because of th. non-locality of $G$ depends on at least one other Integration also Involving a G. As a result, the entire integration falls off faster then $\mathrm{r}^{-3}$. This is illustrated in the second term in (24c) which is proportional to

$$
\iint \mathrm{d} \underline{\mathrm{r}} \underline{\mathrm{d}} \underline{\underline{\prime}}\left[\mathrm{P}_{2}\left(\underline{\underline{r}}, \underline{\underline{r}}^{\prime}\right)-\mathrm{P}_{1}(\underline{r}) \mathrm{P}_{1}\left(\underline{\underline{r}}^{\prime}\right)\right] \int \mathrm{d} \underline{\underline{r}}^{\prime \prime} \mathrm{G}\left(\underline{\underline{r}}, \underline{\underline{r}}^{\prime \prime}\right) \mathrm{G}\left(\underline{\underline{x}}^{\prime \prime}, \underline{\underline{r}}^{\prime}\right)
$$

In light of the above remarks, I belleve an examination of the $\Sigma(t)$ to all orders Indicates that $e^{*}$ is well-defined, $i^{e}$. shape independent. The key is the abllity of sum $(I+G\langle T\})^{-i}$ to subtract out the conditionally convergent terms in $\langle T\rangle$ on a one-to-one basis. The expansion of $\Sigma$ in cumulants is a convenient, but not necessary, vehicle to discuss this. If (17) was the basis for defining $e^{*}$, which essentially is Batchelor's basis, $\epsilon^{*}$ would be inherently shape dependent and hence 111-defined.

One should note that implicit in the above discussion 18 the need for caution in approximating (13). The CPA, (18), is directly expressible as a sum of corrected cumulants and thus is expected to be a shape Independent approximation.

Afmittedly, I have not presented a proof; a proof has been developed by Baker. 43 My inteation was to be heuristic. The question of improperly convergent terms in the perturbation series has gone unnoticed for nearly eighty years and the existence of these difficulties is still unknown to many interested in the effective behavior of heterogeneous materials. I myself just heard of the difficulties several weeks ago. Hopefully, the problems now have the attention and Interest of a wider community.

\section{ACKNOWLEDGRIENTS}

This work was perforned under the auspices of USERDA. The first part of this paper was adapted from a presentation made at the Second International Symposium on Continuum Models of Discrete Systems (Mont Gabriel, Quebec, June 26 - Juiy 2, 1977). This presentation beneflted from helpful conments by J. A. Krumhansl and R. Silver. It was at the Symposium I first heard of the apparent problems of conditionally convergent integrals. (The interested are 
referred to papers by $D$. J. Jeŕfery and $J, R$. Willis in the proceedIngs of that meeting.) In th1s paper the section on conditionally convergent Integrals benefited from a helpful discussion with George A. Baker, Jr.

\section{REFEREACES}

1. G. K. Batchelor, J. F1uid Mech,, 52, 245 (1972).

2. G. R. Batchelor and J.. T. Green, $\overline{56}, 401$ (1972).

3. G... K. Batchelor, Ann. Rev. Fluid Kech., 6, 227 (1974).

4. J. C. Maxwell, Treatise on Blectricity and Magnet1sm, (Oxford Press, Oxford), P. 365.

5. J. C. Maxwell-Garnett, Phil. Trans. R. Soc., 203, 385 (1904).

6. J. C. Maxwel1-Garnett, Phil. Trans.. R. Soc., 205, 237 (1904).

7. D. A. de Vries, Int. Inst. of Refrigeration, (Annexe 1952) 32, 115 (1952).

8. R. J. Elliott and D. W. Taylor, Proc: R. Soc. Lond. A, 296, 161 (1967).

9. E. Kröner, J. Mech. Phys. Solids, 15, 319 (1967).

10. R. J. Elliott, J. A. Krumhansl, and P. L. Leath, Rev. Mod. Phys., 46,465 (1974).

11. F. Yonezawa and K. Morigaki, Prog. Theor. Phys. Supp1., 53, 1. (1973).

12. D. A. G. Bruggeman, Annln. Phys., 24, 636 (1952).

13. R. A. Landauer, J. Appl. Phys., 23, 779 (1952).

14. A. V. Hershey, J. App1. Mech., 21, 236 (1954).

15. R. J. Hill, J. Mech. Phys. Solids, 13, 89 (1965).

16. B. Budlansky, J. Mech. Phys. Sol1ds, 13, 213 (1965).

17. D. W. Taylor, Phys. Rev., 156, 1017 (1967).

18. P. Soven, Phys. Rev., 156, 809 (1967).

19. M. Hor1, J. Math. Phys., 14, 514 (1973).

20. M. Hor 1, J. Math. Phys., 14, 1942 (1973).

21. M. Hol' 1 and F. Yopezawa, J. Math. Phys., 15, 2177 (1974).

22. M. Horl and F. Yonezawa, J. Math. Plys., $\overline{16}, 352$ (1975).

23. M. Hor1, J. Math. Phys., 16, 1777 (1975).

24. M. Hori, J. Math. Phys., 18,487 (1977).

25. M. Hor 1 and F. Yonezawa, J. Phys. C, 10, 229 (1977).

26. E. Domany, J. E. Gubernat18, and J. A. Krumhans1, J. Geo. Res., 80,4851 (1975).

27. J. E. Gubernatis and J. A. Krumhansl, J. Appl. Phys., 46, 1875 (1975).

28. W. F. Brown, Jr., J. Chem, Phys., 23, 1514 (1955).

29. D. Stroud, Phyo, Rev. B, 12, 3368 (1975).

30. R. E. Smith, G. B. Spence, J. E. Gubernatis, and J. A. Krumhane1, Carbon, 14, 185 (1976).

31. D. M. Wood and N. W. Ashcroft, Phil. Mag., 35, 269 (1977).

32. P. H. Dedertchs and R. Zeller, Z. Phys., 259, $103(19 / 3)$.

33. J. Rorringa, J. Math. Phys., 14, 509 (1975).

34. S. K1rkpatrick, Rev. Mod. Phys., 45, 574 (1973).

35. W. L. Bragg and A. B. P1ppard, Acta. Cryst,; 6, 865 (1953).

36. J. Bernescon1, Phys. Rev. B, 9, 4575 (1974).

37. J. Bernascont and H. J. Wiesmann, Phyo. Rev. B, 13, 1.131 (1976). 
38. M. Beran, J. Appl. Phys., 39, 5712 (1968).

39. R. S. Smith, J. App1. Phys., 27, 824 (1956).

40. Y. Yuge, J. Stat. Phys., 16, 339 (1977).

41. D. J. Jeffery, Proc. R. Soc. London A, 335, 355 (1973).

42. D. J. Jeffery, Proc. R. Soc. London A, 338, 503 (1974).

43. J. R. Willis and J. R. Action, Q. J. Mech. Appl. Math., 29, 163 (1976).

44. G. A. Baker, Jr., unpublightd. 